\title{
Understanding Educational Games Culture in Extending the Relationship between Graphic Appearance and Experiences among Malaysian Lower Secondary
}

\author{
Lilian Lee Shiau Gee ${ }^{1,1}$ Jasni Dolah ${ }^{1}$, and Norfarizah Mohd Bakhir ${ }^{1}$ \\ ${ }^{1}$ School of the Arts, Universiti Sains Malaysia, 11800, Malaysia
}

\begin{abstract}
Educational games provide a virtual environment for the students, particularly in an endeavour to increase student immersion, and this need of students satisfied with the visual aspect conditions of the game screen to go to the following stage. In determining the effect of visual design on students' learning experiences - it's required a further analysis within the students' motivation, engagement and enjoyment. To achieve this goal, a solid fundamental understanding in theoretical philosophy, which closely related to describe the relationship of visual experience, especially in intrinsic tendencies theory and visual principles that on holds the context of motivation, engagement and enjoyment among students. In this regard, a thorough discussion of relevant theories were led to the suggestion theoretical framework and proposed of Experience Based Design Graphic Style (EBDGS) to identify student's experiences (motivation, engagement and enjoyment) outcomes which mostly depends on the visual design approaches to designing the game scenarios. This paper also aims to describe the theoretical framework proposed in understanding the Malaysian student experience's outcomes by placing the abstract style and realistic style in developing Experience Based Design Graphic Style (EBDGS) game prototype: EBGDS A (Abstract) and EBGDS R (Realistic). The implication of the theoretical framework was focused on the visual design (graphic styles) of games that intentionally centred in the students' experience that tied to the demographic factors (gender, type of schools and areas), and the resulting experiences are examined through the Student Experience in Graphic Measurement Scale (SEIGMS). The intention of this research not only emphasize the suitability visual appearance able better improve student experiences but also increases deeper local knowledge in visual design guidelines in educational game design contexts.
\end{abstract}

Keywords : Educational Games, Graphic Styles, Students' Experiences

\section{Introduction}

Lin et al [1] argued that educational game are enacted by students, and the students were also become enacted by the gaming environment, which the design should be constituted with a better visual to enable students to prescribe, sustain and generate personal experiences, onwards increasing continuous in the learning process. In this way, efficiency educational games usage needs overviewed in terms of visual design features that may conducted student's attentiveness $[2,3,4,5,6]$.

In Malaysia, the implementation of educational games is still in an early stage [7] with concerned the development and evaluation the design context of educational games for enhancing instruction and learning conditions in the classroom. With regards, the preliminary investigation indicating the development of an educational game that comes with the learning approach process needed take into consideration the design context in visual design aspects to arouse the students' interest especially for local Secondary School students. Moreover, most previous studies not centered on

\footnotetext{
${ }^{1}$ Corresponding author: lilianlsg087@gmail.com
} 
the efficiency educational games usage in the visual design aspect in the suitability of the visual design on student experiences. Additionally, lack of exposure an organized design technique or a style, including less established basic design guidelines to assist designers and educators make an understanding and skills to the concept's suitability of visual design in educational game development on student experiences.

With the lack of empirical studies or incentive information to distinguish the purpose of design (visual aspect) that needs to be adapted in developing educational games in improving the good experience among students. Therefore, this study needs to be carried out to identify how visual design important to shape usability of educational game in enhancing the individual students' affective responses-by taking a closer look at the continually experience outcomes, particularly in terms of motivation, engagement and enjoyment. Thus, this study also explores consistencies intended student experiences by mapping the "visual" details and knowledge aspects of local student culture - as assessment of the degree efficiency educational games towards the ways of appearance. Further, understanding the relationship between graphic appearances and student experiences becomes relevant in an attempt to studying gaming scenarios in accord to factors of demographic that involves the complementary experiential aspects.

\section{The Relation of Visual Design and Experiences}

In intended that any design for educational games (design-in-use) should concerned with suitability visual appearances (design intentions) by prioritizing students' reactions (experiences), which in relation to local student culture context. This approach the term "graphic styles" as the key analytical concept in discussing and analysing the role of educational games, which verity the relationship between the visual design intentions and local student preferences as closely tend to sustain the local practices (teaching and learning) - also important tries to understand the visual design specifications align with the student's interests, and developing subject-related knowledge in local classroom contexts in relation local culture (demographic factor). In this respect, the demographic factor assumes a close related to student gender, educational area and types of schools.

Correspondingly, the value of visual design to the educational gaming environment is linked to the actual student experiences changes, in relation what kind of graphic styles be able to enhancing and maintaining students experiences and recommendable to developers. Thus, the visual design is valued to be regarded as an effort to understand the hedonic experiences turn in educational game development, which emphasis on students adapt and interact with particular visual representation in relation to their own experience practices and preference criteria.

\section{Related Theories}

\subsection{Motivation Theory}

As indicated by Keller [8] "motivational tactics have to support instructional goals", which shows that motivation theory impact on the instruction development and closely linked to maintaining the students' interest in teaching and learning process. Keller was founded four main elements in the learning process that can motivate students or known as ARCS model. The four elements that built the ARCS model stand out Attention, Relevance, Confidence and Satisfaction in growing student's interest in an instructional tool, in relation to understanding student experiences and instruction design.

\subsection{Self-Determination Theory}

The advances of motivation may restructure an importance experiences impact in educational games. From students' experiences, there is an urgent need to understand the relationship interplay between cognition, motivation and feelings in a process of learning through educational games. In this, the Self-Determination Theory (SDT) becomes the foundation for researchers to acquire, analyse and evaluate the players' experience in games [9]. As a supporter of motivation, Self-Determination Theory implicated three basic psychological needs (competence, autonomy and relatedness) are itemized to identify motivation value in a digital game [10]. Understanding the Self-Determination Theory able to practice assessing the quantity of experiences that could take place in educational games in the appraisal of the significant experiences through the game design features and actors (students) personal complacency. 


\subsection{Visual Attention}

Visual attention actively sustained concentration to a certain number based on the visual object influence the ongoing action and cognition [11]. For enhance a person's ability to recognize certain visual concepts in learning and instruction, visual perception process and experience are intimately related [12]. As added by Chun et al [13], visual attention is an active maintenance of visual processing towards visual items to enhanced ongoing experience. Accordingly, visual attention emphasized knowing individual's experience through the selection of visual features that should attend in learning activities that can clarify the efficiency of visual to delivering knowledge. This also indicates the importance of visualization in a game to attract the concentration of the target audience.

\subsection{Gestalt Theory}

Gestalt theory describes the significance of visual elements in an object to increase the individual's reception and tendency to attract a person's attention in a certain visual presentation [14]. Instead, this theory function as the suggestion aspect and practicing various visual elements (shape, form and composition) should be presented to achieve efficient visual image results [15]. Thus, Chang and Nesbitt [16], suggested the conception of applying the gestalt principles to assist the visual presentation in games, which is necessary understanding the gestalt principles in producing a visual image in game scene design in order to encourage players' involvement.

\subsection{McCloud Theory}

McCloud [17] presents a basic theory of icon (a visually image resembling the real object of several aspects). According to McCloud, a continuum from realistic to cartoon images showed increased levels of abstraction iconic. These changes simplify the image of visibility. In addition, McCloud theory distinguishes identification image into three main categories: abstraction, cartoony, and realistic. Thus, understanding the concept of imagery increasing, convenience construct the visual appearances in an educational game.

\section{Graphic Styles}

The word 'style' in the field of visual art related to the composition and the relationship between the form an overall image, that based on the principle elements, techniques, expression and inspiration [18]. According to Muller [19], graphic style refers as an implementation technique that carried out by artists to portray the mood and features to be conveyed on the audiences. This is simply integrated that graphic display in game are more delineate on surrounding representations and components or objects that are placed in the game environment to enhance gameplay, which the graphic styles can be ranged from abstract to realistic [20]. As this, McLaughlin et al [2] describes the graphic styles are categorized into three different types: i) abstract, ii) stylized and iii) realistic. Each graphic style has been summarizes (see Table 1) as accordance with the form, surface and level of detailed.

Table 1. Description of Graphic Styles (McLaughlin et al, 2010)

\begin{tabular}{|c|c|}
\hline Abstract & $\begin{array}{l}\text { - Symbolic forms. } \\
\text { - Reducing the formal quality } \\
\text { - Low in the level of detail } \\
\text { - } \quad \text { Flat Shadings }\end{array}$ \\
\hline Stylized & $\begin{array}{l}\text { - Identifiable forms (unrealistic proportions or juxtapositions of } \\
\text { parts) } \\
\text { - } \text { Quality is expressive } \\
\text { - The level of detail can be composed from the lower to higher } \\
\text { - Shading of curved surfaces or have a texture }\end{array}$ \\
\hline Realistic & $\begin{array}{l}\text { - Presentation style in photo-realistic modelling accurately the } \\
\text { familiar objects. } \\
\text { - Level of detail is high. } \\
\text { - Realistic shadow casting, Light movement }\end{array}$ \\
\hline
\end{tabular}




\section{Student Experiences}

In addition to theories of learning and visual knowledge, experiences viewed as important facets that is prized for the ability, suitability and efficiency of educational games within the student's interest. In this regards, student experiences will be reviewed in this research were based upon the motivation, engagement and enjoyment to further examining the influence of visual design in educational gaming, which assume a relational meanings and local knowledge aspect of educational game competences by actively engaging the visual design aspect that distributed particular relationships between the student preferences of a visually style gaming.

\subsection{Motivation}

In games, motivation playing an important function in gaming experiences - as a process of activating, maintaining the player behaviour towards a certain goal, though personal interest and the way player organizing the experiences in gaming [21]. In this way, motivation able pushed the players becoming more engagement and involved with games and turn the games be more successful [22]. With this regard, the relationship between students' experiences with game more domain considering intrinsic motivation to analyse the motivating factors [23]. This means that, the potential usage of educational games need be viewed especially on student experiences in terms of intrinsic motivation.

\subsection{Engagement}

In games, engagement viewed as the player's degree of involvement in a digital games to attract player's continuous play [24]. Thus, engagement is one of the most frequently cited and needed to consider in digital game for enhancing the student experiences [25]. This indicated that engagement experience is a connector between the students emotional emerged between educational games- that also relies on the educational game design, particularly game features to preserve the degree of interests [26].

\subsection{Enjoyment}

Enjoyment is in respect of individual's perception of pleasant feeling when conducting an activities [27]. As such, enjoyment regarded as an important source of experience in student learning [28]. Generally, enjoyably viewed as an experience strictly related to motivation and the factors in facilitating the engagement in software application [29]. This resulting enjoyment experience was interlink with motivation and engagement, which need take into consideration in determining the suitability of educational game appearances.

\section{Theoretical Framework Overview}

In order to identify and analyse the students' experience outcomes throughout interplay between students interaction and visual design contexts, the theoretical framework draws upon with the adoption of Activity Theory (AT) that conducted by Engestrom [30] in 1993. The Activity Theory review the contexts between human (subject) and their reacts upon (object) on a tool that concerned an individual responds, which based on the tools that occur during the reception process of information [31]. With compliments to the theory, the subject replaced with students, the object is substituted with the experiences and the tool replaced with graphic styles - to clarify what experiences result could have within the visually contexts. The Activity Theory system for describing the relationship between graphic styles and students are depicted in Figure 1.

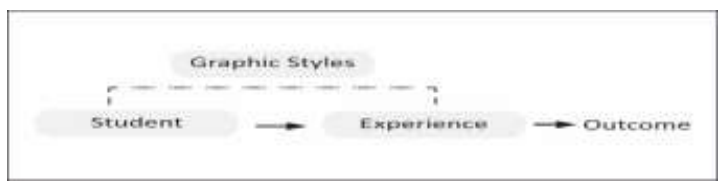

Fig. 1. Structure of Activity Theory 
Correspondingly, the theoretical framework was introduced into macro and micro level (see Figure 2). Following the principles of Activity Theory, the macro level tries to indicate the student experiences by viewing students' preference and students demographic as the distinct criteria knowledge for arises local knowledge in game design practices. Simply applied, realistic style and abstract style represent different assumptions about the outcomes of student experiences through different ways of enacting within the demographic factors, which would be making of meaning at the local level of visually context within local student preferences and local syllabus content. Micro level is more concretely in development of Experience Based Design Graphic Style (EBDGS) prototype - a continuum learning activities, game characteristics and visual design represent modes with the intent to understand the student experiences encounter as they emerge through students' enactment of a particular style of educational game scenarios.

This approach to explore the lower secondary students (Form One student) make meaning from educational games in the context of visual display through the ways they enacted and validated their experiences by facilitating and performing within the EBDGS game prototypes: EBDGS A (Abstract) and EBDGS R (Realistic). In this way, Student Experience in Graphic Measurement Scale (SEIGMS) questionnaire are present to generalised the local student view on their motivation, engagement and enjoyment outcomes within the consideration of graphic style quality in designing educational gaming. Thus, students can be seen as "social actors" are free to play and interact with different delivery visual representation modes (abstract or realistic), based on their experience's result outcomes is important to distinguish the substantive quality of visual appearances that imply an interpretive guideline to enhance local student involvements in learning and opportunities for local educational game development.



Fig. 2. Theoretical Research Framework

\section{Conclusion}

This paper explores a theoretical framework for educational game design comprising the graphic styles, demographic factor and students' experiences domain as for understanding the local student perspectives in order to emerge the local knowledge of the educational game development in visual design context. The related theories and key literatures have provided a rich theoretical basis for the theoretical framework development with imply the challenges and issues that inherent incongruities between the game design (which prioritises visual design) in relation to the mode of representation (graphic styles), demographic factor and the students' experiences. In this respect, the relation between particular graphic appearance and students' experiences needs to be determined, for identifying new views on visual design trends that should embedded in educational games, as an external factors that may have influences on local 
perceptions (students). Hence, the implementation of EBDGS A (Abstract) and EBDGS R (Realistic) is to determine the student experiences by viewing at the graphic style and the experience outcomes of play activities with the aim of understanding the designing concept in increasing the educational game's efficiency without discrediting other current research efforts on understanding educational games. This research assumes that empirical findings were possible capable to accomplish further the local contexts (foreground and background) that tries to formulating the visual design guideline for the local designers and educators to offering an efficient learning presentation and organize a strategic plan enhancing the educational game appearances in advance.

\section{References}

1. Lin, Y.L., Lin, H.W. and Yang, Y.T. (2015). Players' Value Structure in Digital Games.

2. Games and Culture, 1-28.

3. McLaughlin, T., Smith, D., and Brown, I.A. (2010). A framework for evidence based visual style development for serious games. Proceedings of the Fifth International Conference on the Foundations of Digital Games, ACM (2010), 132-138.

4. Andersen, E., Liu, Y., Snider, R., Roy, S., Zoran, P. (2011). Placing a Value on Aesthetics in Online Casual Games. In: ACM CHI Conference on Human Factors in Computing Systems.

5. Carroux, L., Le Bigot,L. Vibert,N. (2013). Impact of the motion and visual complexity of the background on players' performance in video game-like displays. Ergonomics, 2013;56(12):1863-76. Doi: 10.1080/00140139.2013.847214. Epub2013 Oct 30.

6. Gerling, K. M., Birk, M., Mandryk, R. L., \& Doucette, A. (2013). The Effects of Graphical Fidelity on Player Experience. MindTrek, 229-236.

7. Wang, L., \& Lee, K. (2014). The Study Of Learners' Preference For Visual Complexity On Small Screens Of Mobile Computers Using Neural Networks, Tojet: The Turkish Online Journal Of Educational Technology 13(2), 168-180.

8. Roslina, I. \& Azizah, J. (2009). Educational Games (EG) Design Framework: Combination of Game Design, Pedagogy and Content Modeling. 2009 International Conference on Electrical Engineering and Informatics 5-7 August 2009, Selangor, Malaysia.

9. Keller, J. M. (2006). Motivation and performance. In R. A. Reiser \& J. V. Dempsey (Eds.), Trends and Issues in Instructional Design and Technology (2 ed., pp. 82-92). Upper Saddle River, NJ: Merrill/Prentice Hall

10. Bouvier, P., Lavoué, E., Sehaba, K., \& George, S. (2013). Identifying learner's engagement in learning games: A qualitative approach based on learner's traces of interaction. In O. Foley, M. T. Restivo, J. O. Uhomoibhi, \& M. Helfert (Eds.), Proceedings of the 5th International Conference on Computer Supported Education (pp. 339-350). SciTePress: Aachen, Germany.

11. Uysal, A., \& Yildirim, I. G. (2016). Self-Determination Theory in Digital Games. Gamer Psychology and Behavior, 123-135.

12. Chun, M. M. (2011). Visual working memory as visual attention sustained internally over time. Neuropsychologia, 49(6), 1407-1409.

13. Silvia, P. J. (2001). Interest and interests: The psychology of constructive capriciousness. Review of General Psychology, 5(3), 270-290.

14. Chun, M. M., Golomb, J. A., \& Turk-Browne, N. B. (2011). A taxonomy of external and internal attention. Annual Review of Psychology, 62, 73-101.

15. Koffa, K. (1935): Principles of Gestalt Psychology. London, Routledge and Kegan Paul.

16. Chang, D., Dooley, L., \& Tuovinen, J. E. (2002). Gestalt Theory in Visual Screen Design - A New Look at an Old Subject. In Proc. WCCE2001 Australian Topics: Selected Papers from the Seventh World Conference on Computers in Education, Copenhagen, Denmark. CRPIT, 8. McDougall, A., Murnane, J. and Chambers, D., Eds. ACS. 5-12.

17. Chang, D. and Nesbitt, K. (2006). Identifying Commonly-Used Gestalt Principles as a Design Framework for Multi-Sensory Display, Proceedings of IEEE International Conference on SMC, Taipei, Taiwan.

18. McCloud, S. 1994. Understanding Comics: The Invisible Art.

19. Dondis, D.A. A. (1973). Primer of Visual Literacy. MIT Press Design Department. United States of America. 
20. Muller, J. (1979).Structural studies of Art Styles. In Cordwell, J.M. (Ed.), The Visual Arts: Plastic and Graphic. 139-212. Mouton Publishers. Great Britain.

21. Frohardt, D. (1999). Teaching Art with Books Kids Love: Teaching Art Appreciation, Element of art, and Principles of Design with award-Winning Children's Books. Fulcrum Publishing. United States of America.

22. Bragg, L. A. (2003). Children's perspectives on mathematics and game playing. In L. Bragg, C. Campbell, G. Herbert \& J. Mousley (Eds.), Mathematics education research: Innovation, networking, opportunity (Proceedings of the 26th annual conference of the Mathematics Education Research Group of Australasia, Geelong, (Vol. 1, pp. 160-167). Sydney: MERGA.

23. Scoresby J., Shelton B. (2011). Visual perspectives within educational computer games: Effects on presence and flow within virtual immersive learning environments. Instructional Science, 39, 227-254.

24. Medina, E. (2005), Digital Games: A Motivational Perspective, Proceedings of DiGRA 2005.

25. Brown, E., \& Cairns, P. (2004). A grounded investigation of game immersion. In CHI '04 Extended Abstracts on Human Factors in Computing Systems (pp. 1297-1300). New York, NY: ACM.

26. Bouvier, P., Lavoue, E., \& Sehaba, K. (2014). Defining Engagement and Characterizing Engagement Behaviors in Digital Gaming. Simulation \& Gaming, 45(4-5), 491-507.

27. Rozendaal, M. C., Keyson, D. V., de Ridder, H., \& Craig, P. O. (2009). Game feature and expertise effects on experienced richness, control and engagement in game play. AI and Society, 24(2), 123-133.

28. Barnett, L. A. (1990) Playfulness: Definition, Design, and Measurement. Play and Culture, 3, 319-336.

29. Lumby, J. (2011). Enjoyment and learning: Policy and secondary school learners' experiences in England. British Educational Research Journal, 37, 247-264.

30. Abdul, A. \& Felicia, P. (2015). Gameplay Engagement and Learning in Game-Based Learning A Systematic Review. Review of Educational Research

31. Engestrom, Y. (1993). Developmental studies of work as a test bench " of activity theory: The case of primary care medical practice. In S. Chaiklin \& J. Lave (Eds.) Understanding practice: Perspectives on activity and context (pp. 64-103). Cambridge, MA: Cambridge University Press

32. Squire, K. D. (2002). Video Games and Education: Designing learning systems for an interactive age. 